\title{
El papel de los organismos no estatales de estandarización en la formulación del Marco Internacional de Información Integrada*
}

\author{
The role of non-state standardization bodies in the formulation of the International Integrated \\ Reporting Framework \\ O papel dos organismos de normalização não estatais na formulação da Estrutura Internacional do \\ Relato Integrado
}

\author{
Yaismir Adriana Rivera-Arrubla ${ }^{\text {a }}$ \\ Universidad del Valle, Colombia \\ yaismir.rivera@correounivalle.edu.co \\ ORCID: https://orcid.org/0000-0002-0340-2019
}

DOI: https://doi.org/10.11144/Javeriana.cc22.poee

Recibido: 04/07/2020

Aceptado: $21 / 12 / 2020$

Publicado: $18 / 08 / 2021$

\section{Resumen:}

En los últimos años el Marco Internacional para la Información Integrada ha sido promovido como la norma global de información corporativa, y avanza en el espacio regulador, gracias al apoyo de sus fundadores y a la evolución previa de otros estándares internacionales. En este artículo analizamos las cartas de comentarios dirigidas por organismos de estandarización no estatal, participantes del proyecto de consulta del marco, cuya posición estratégica influyó el contenido publicado por el IIRC en 2013. En esta tarea identificamos el impacto de sus posiciones frente a la definición de un marco basado en principios, el concepto de IR y el público destinatario del informe. Nuestras conclusiones destacan que estos organismos, y en general, los procesos de estandarización privada tienen gran influencia en la definición de estándares esenciales tanto para este informe como para la evolución de las prácticas de rendición de cuentas. Las instituciones analizadas influyeron en los contenidos del marco, ocuparon roles relevantes en el gobierno y fuerzas de trabajo del IIRC y continúan trabajando en que esta tendencia se abra paso a partir de su experiencia, alianzas y recursos.

Código JEL: M14.

Palabras clave: Información integrada, Marco Internacional de Información Integrada, organismos de estandarización no estatal, legitimidad, debido proceso.

\begin{abstract}
:
In recent years the International Integrated Reporting Framework has been promoted as the global standard for corporate reporting and advances in the regulatory space, thanks to the support of its founders and the evolution of previous international standards. In this paper we analyze the comment letters addressed by non-state standardization bodies, who participanted in the framework consultation project, and whose strategic position influenced the content published by the IIRC in 2013. For this, we identify the impact of their positions regarding the definition of a principles-based framework, the concept of IR, as well as the target audience for this report. Our conclusions highlight that the analyzed agents, and in general, the processes of private standardization, have a great influence on the definition of essential standards, both for this report and for the evolution of accountability practices. The institutions analyzed influenced the contents of the framework, occupied relevant roles in the IIRC government and work forces, and continue to work through their experience, alliances and resources to make this trend successful.
\end{abstract}

JEL Code: M14.

Keywords: Integrated reporting, International Integrated Reporting Framework, non-state standardization bodies, legitimacy, due process.

\section{Resumo:}

Nos últimos anos a estrutura internacional para relato integrado tem sido promovida como o padrão global de informação corporativa, e avança no espaço de regulação, graças ao apoio de seus fundadores e à evolução anterior de outros padrões internacionais. Neste artigo analisamos as cartas-comentários dirigidas por organismos de normalização não estatais, participantes do projeto de consulta da estrutura, cuja posição estratégica influenciou o conteúdo publicado pelo IIRC em 2013. Nessa tarefa, identificamos o impacto de seus posicionamentos na definição de uma estrutura baseada em princípios, no conceito de IR e no público-alvo do relatório. As nossas conclusões destacam que estes órgãos, e em geral os processos de normalização privada, têm

Notas de autor

a Autora de correspondencia. Correo electrónico: yaismir.rivera@correounivalle.edu.co 
grande influência na definição dos padrões essenciais tanto para este relatório como para a evolução das práticas de prestação de contas. As instituições analisadas influenciaram os conteúdos da estrutura, ocuparam papéis relevantes no governo e nos grupos de trabalho do IIRC e continuam trabalhando para que essa tendência se estenda a partir de sua experiência, alianças e recursos.

Código JEL: M14

Palavras-chave: Informações integradas, Quadro Internacional de Informações Integradas, organismos não-estatais de padronização, legitimidade, devido processo.

\section{Introducción}

El Marco Internacional de Información Integrada (IIRF por sus siglas en inglés) tiene como propósito guiar las prácticas de implementación del Informe Integrado alrededor del mundo (IIRC, 2012a). Su desarrollo está a cargo del International Integrated Reporting Council (IIRC), institución fundada en 2010 y dirigida por destacados actores en el campo de la estandarización privada (Rowbottom \& Locke, 2016). Entre sus miembros, se cuentan importantes líderes mundiales representantes del sector corporativo, de inversión, contable, valores, regulatorio, académico y de la sociedad civil (IIRC, 2013a, 2013b).

Si bien, las prácticas de Información Integrada (IR por sus siglas en inglés) fueron emprendidas por iniciativa de algunas empresas a partir del año 2000, es decir, una década antes de su introducción oficial, sólo a partir de la fundación del IIRC, la IR es presentada como un movimiento que pretende comunicar el rango completo de factores que afectan la habilidad de una organización para crear valor en el tiempo (IIRC, 2013b). Su propósito es superar la fase de presentación de numerosos informes desvinculados y estáticos (IIRC, 2013b), permitiendo entregar una imagen completa de las organizaciones a corto, mediano y largo plazo. Lo que significaría corregir las limitaciones propias de los informes financieros y de sostenibilidad hasta entonces presentados de forma independiente e inconexa (Eccles \& Krzus, 2010; Jensen \& Berg, 2012; Abeysekera, 2013; IIRC 2013b; AICPA, 2014; Camilleri, 2018).

A pesar de haber sido establecido tras la Mesa Redonda celebrada por el Parlamento Europeo en abril de 2009, en 2013, el IIRC presentaba a la comunidad empresarial y a las partes interesadas, la primera versión del marco IIRF. Es decir, aún pese a atravesar una fase en la que tanto la madurez del concepto como el marco estaban en sus primeras etapas (Rowbottom \& Locke, 2016, Paternostro, 2020), este movimiento era promovido como el futuro de la información empresarial; al tiempo, que el IIRC definía como objetivo ganar suficiente dominio en el espacio regulador para alcanzar la adopción de la IR como norma de información corporativa global (IIRC, 2012a, 2013a, 2013b).

En el desarrollo del marco de referencia, el IIRC afirma haber seguido un debido proceso con un enfoque inclusivo y basado en el mercado que atiende a las necesidades de las empresas, los inversores y la sociedad civil (IIRC, 2013b). Por su parte, el IIRF es un recurso construido socialmente, necesario para la definición de procesos corporativos pensados - al menos inicialmente- bajo formas de creación de valor sostenible, que orienten la preparación de informes en ambientes de divulgación tanto voluntaria como obligatoria.

Dada su naturaleza revisable y su concepción basada en principios (IIRC, 2013a, 2013b) se esperaría que el IIRF fuese un medio flexible que traduzca en reglas las necesidades de distintos agentes, ordenando las estructuras organizacionales (Rowbottom \& Locke, 2016). De hecho, la concepción del IIRF tendría consecuencias prácticas que ameritan su estudio, ya que en la medida que este marco consiga establecerse y ser aceptado como norma logrará introducirse en la estructura de las prácticas de rendición de cuentas, dominando así las tendencias y patrones de preparación y presentación.

Con el propósito de analizar algunos antecedentes del marco, y en particular, la acción e influencia de sus promotores, este artículo tiene como objetivo estudiar a la coalición IIRC como un producto de la experiencia e intereses de una red de actores estratégicos, y en particular, analizar los desarrollos previos de otros organismos reguladores de carácter no estatal, con amplia trayectoria en materia de estándares corporativos. De hecho, este marco corresponde a la evolución de estándares, que atañen tanto a la dimensión 
financiera como a contenidos sociales y medioambientales diseñados principalmente a partir de bases no obligatorias (de Villiers, Rinaldi, \& Unerman, 2014; de Villiers, Hsiao, \& Maroun, 2017).

Otros estudios han analizado el proceso de lobbying seguido por el IIRC para institucionalizar el IIRF (Oprisor, 2014, Simnett \& Huggins, 2015, Reuter \& Messner, 2015); no obstante, la literatura relacionada con la IR, y en general con estándares corporativos, se ha basado principalmente en corrientes positivas y económicas, descuidando otros enfoques y perspectivas. En su lugar, el planteamiento de base en esta investigación parte de considerar que el IIRF ha sido desarrollado en un contexto de estandarización dominado por organismos trasnacionales de carácter privado, por lo que corresponde a un fenómeno político cuya legitimidad depende de la aceptación de sus constituyentes así como de su posición estratégica (Giddens, 1984). Fenómeno presente en los últimos años, en el que el poder de los organismos a cargo de los procesos de estandarización, no reside en la soberanía política tradicional, sino en la legitimidad otorgada por las audiencias interesadas, entre ellas, el estado (Suchman, 1995; Cashore, 2002; Richardson \& Eberlein, 2011).

Este artículo tiene un doble propósito: el primero, poner de relieve la influencia que la regulación privada tiene hoy por hoy, sobre proyectos fundamentales de información corporativa, como lo es el establecimiento de las bases y principios de la IR. Para esto se revisa cómo a través de un proceso de consulta pública algunos organismos no estatales consiguieron tener un papel destacado en la configuración del IIRC y en la definición del IIRF. El segundo objetivo es analizar la participación de destacados organismos estandarizadores como The American Institute of Certified Public Accountants (AICPA), Global Reporting Initiative (GRI), International Federation of Accountants (IFAC) y Sustainability Accounting Standards Board (SASB), a partir de estudiar las cartas de comentarios enviadas por estos agentes durante la consulta hecha por el IIRC en 2013. El procedimiento empleado consiste en revisar sus respuestas, contrastando la influencia de estos organismos sobre el contenido del marco finalmente publicado. Con este propósito identificamos sus posiciones respecto de los siguientes aspectos clave en la estructura de la IR y el IIRF, que constituyen nuestro objeto de estudio: (1) la definición de un marco basado en principios, (2) el concepto de Información Integrada y (3) la demarcación del público destinatario del informe.

\section{El papel de los organismos no estatales en el desarrollo de estándares de información no financiera}

La tarea de emitir estándares y asegurar su cumplimiento solía estar en cabeza de organismos gubernamentales con suficiente autoridad para imponer la aplicación de normas a partir de un ejercicio de soberanía. Actualmente, este rol no se limita a la acción estatal (Cashore, 2002; Suarez, 2004; Peters et al., 2009a, 2009b), podría decirse incluso, que cada vez con mayor efectividad y extensión de aquella atribuible al estado, la regulación no estatal, juega un papel decisivo en la definición de parámetros sobre un determinado conjunto de estándares (Suarez, 2004; Bartley, 2005, 2007; Suddaby, Cooper, \& Greenwood, 2007; Vogel, 2008; Sikka, 2008; Bendell, Miller, \& Wortmann, 2011; Richardson \& Eberlein, 2011), más cuando se trata de aquellos de tipo no financiero (Camilleri, 2018).

Por organismos no estatales se entiende entidades o asociaciones de carácter nacional, internacional y supranacional, organizaciones no gubernamentales (ONGs), standard-setters y cuerpos profesionales. El contexto que ha servido para la proliferación de estos agentes se atribuye a la vez a procesos de privatización, liberalización y globalización acaecidos entre las décadas de 1980 y 1990, los cuales condujeron, además, a la integración de economías nacionales en lo que hoy se denomina como economía global (Peters et al., 2009a).

Este fenómeno ha sido ampliamente estudiado por la literatura académica relacionada con la producción de estándares de información corporativa. Además, está conectado a las ciencias políticas que estudian, tanto la naturaleza y características de los sistemas de gobierno no estatales, como los problemas alrededor de 
la autoridad y legitimidad de estándares producidos bajo iniciativas privadas (Cashore, 2002; Beisheim \& Dingwerth, 2008; Peters et al., 2009a, 2009b; Richardson \& Eberlein, 2011).

El concepto de legitimidad es clave en este trabajo para comprender los procesos de producción de estándares como actos socialmente construidos bajo la percepción que estos son producto de la coherencia entre los comportamientos de una entidad valorada por el público como legítima y las creencias compartidas por dicho grupo social (sus constituyentes). Se supone entonces, que la legitimidad depende de una audiencia colectiva y no de algunos de sus observadores en particular (Suchman, 1995).

En el campo específico de los informes corporativos, los avances en aspectos no financieros han sido influenciados por la rápida emergencia de estándares internacionales sobre preocupaciones sociales y ambientales de amplio interés social. Y como ocurre con otras guías y marcos de referencia, los reportes relacionados con asuntos de Corporate Social Responsibility (CSR), incluida la IR, suelen estar precedidos por políticas públicas en pro del desarrollo sostenible, defendido como un objetivo político de las naciones y resultado de iniciativas de cooperación intergubernamental. Esto quiere decir, que, pese al interés estatal por estos temas, la proliferación de estándares no obligatorios se atribuye mayoritariamente a acciones emprendidas por organismos privados, traducidas en formas alternativas de informes no financieros (Bendell et al., 2011; Rowbottom \& Locke, 2016).

Como producto de esta actividad regulatoria carente de una dirección unívoca, ejercida a partir del estado, es común observar una superposición de estándares, que, no obstante, versar sobre temas similares presentan desarrollos dispares con sus consecuentes conflictos normativos (Peters et al., 2009a). En las últimas décadas se ha formulado un conjunto de guías y estándares de la mano de organismos privados especializados en el establecimiento de estándares corporativos. Ejemplo de esto es que tanto la creciente utilización de estándares internacionales de información financiera como la institución de distintos marcos para la divulgación de información no financiera estén a cargo de instituciones privadas como el International Accounting Standards Board (IASB) (Richardson \& Eberlein, 2011), GRI, AICPA, SASB, Carbon Disclosure Project (CDP), the World Intellectual Capital Initiative (WICI) y en años recientes del IIRC.

A pesar del carácter institucional en el que se produce la regulación por medios no estatales, la estructuración de las distintas agencias que encabezan las tendencias de evolución de estándares tiene lugar en un contexto de libre comercio, softlaw, globalización y auto-regulación. En tales condiciones, el poder del estado-nación y su papel como regulador tradicional estaría afectado por la intervención del mercado y la continua mutación de sus agentes y formas de acción; al tiempo que distintos actores de la sociedad esperan que las organizaciones puedan auto-regularse y contribuir de manera real a resolver problemas relacionados con el desarrollo sostenible (Cashore, 2002; Bartley, 2005; 2007; Vogel, 2008).

Esto evidencia cómo la legitimación del papel institucional de la regulación privada a través de distintos medios ha reemplazado buena parte de la misión estatal de regular a sus agentes. De este modo, la tarea del estado como regulador local que también debe promover la cooperación intergubernamental en pro de comportamientos sostenibles a nivel global ha perdido lugar frente a las iniciativas de estandarización encabezadas por organizaciones privadas y procesos de integración trasnacional (Bartley, 2007; Bendell et al., 2011; Richardson \& Eberlein, 2011). En consecuencia, los estándares de origen no estatal han alcanzado una relevancia comparable con las normas emitidas por instituciones locales con respaldo de ley (Fortanier, Kolk, \& Pinkse, 2011).

Si bien en los últimos años se han creado y fortalecido diversos organismos de carácter estatal, son las iniciativas privadas las que con mayor efectividad puede atribuirse el desarrollo acelerado de diversos estándares de información no financiera (Bartley, 2007; Nadvi \& Wältring, 2004). Y son precisamente esas iniciativas las que controlan de manera agregada la formación y el alcance de instituciones como el IIRC. Los organismos financiados por contribuciones voluntarias y de origen privado dominan el escenario de estandarización internacional (Nölke \& Perry, 2007). Entre estos, participan de manera destacada los cuerpos de carácter profesional con un papel muy activo en el trabajo de acreditar la probidad de sus miembros y de 
pautar procedimientos de adopción de estándares voluntarios, lo que finalmente determina buena parte de la arquitectura y el contenido de los informes corporativos (Bebbington, Kirk, \& Larrinaga, 2012) y de las tendencias de divulgación.

Producto de la prolífica evolución de estándares para la divulgación de información externa, y dado que se trata de iniciativas cuyas raíces fueron sembradas varios años atrás, la idea de presentar en conjunto todos los contenidos financieros y no financieros, bajo un único informe sólo se discute -al menos de manera oficialtras la institución del IIRC y bajo el concepto IR.

La rápida conformación del IIRC y el corto período de tiempo en que se formuló el marco internacional ha llamado la atención de algunos autores (e.g., Rowbottom \& Locke, 2016; Abhayawansa, 2014; Humphrey, O’Dwyer, \& Unerman, 2017). No obstante, una posible hipótesis para explicar este hecho es que tras la madurez alcanzada por los informes financieros, de gobernanza y sostenibilidad, la IR sería consecuencia lógica del interés por el desarrollo sostenible y la responsabilidad corporativa (Morros, 2016). Caso en el cual estaríamos presenciando una transición natural desde marcos y conceptos previos hacia la integración de estándares de rendición de cuentas que desembocan en la esperada y necesaria IR. Bajo esta concepción podría pensarse que el IIRC es una suerte de heredero natural de los intereses y el trabajo abierto en distintos frentes relacionados con los procesos de rendición de cuentas.

En este sentido, nuestro planteamiento es que el IIRC es una coalición generada a partir de la intersección de recursos y esfuerzos previamente construidos por diversos actores con un amplio conocimiento y experiencia en el contexto estandarizador. De hecho, los grupos implicados en su fundación y funcionamiento tienen intereses comunes en pautar la evolución de la información corporativa extendiendo la vigencia de sus propios estándares, a partir de la pervivencia y éxito del IR.

El IIRC tiene el reto de crear un fuerte impulso global en torno a la IR (Eccles, Krzus, \& Ribot, 2014) y de adquirir legitimidad tanto para asegurar su continuidad institucional como para obtener credibilidad de su entorno. Con la complejidad adicional que sus divulgaciones sean calificables como un mito (Gibassier, Rodrigue, \& Arjaliès, 2018) de difícil asidero, tanto práctico como teórico, ya que la IR es un concepto aún muy controvertido (Paternostro, 2020). La motivación del GRI y el Prince's Accounting for Sustainability Project (A4S) al patrocinar este nuevo organismo emisor en el campo ya densamente poblado de marcos se explicaría por "el fracaso" en intentos anteriores por conseguir la adopción de un modelo único de informe (Brown, de Jong, \& Levy, 2009; Rowbottom \& Locke, 2016). Como resultado, la IR se abre paso, gracias a una estrategia pensada para globalizar un único conjunto de estándares, con el compromiso del IIRC de asegurar el reconocimiento del IIRF entre distintos grupos de interés, organismos de estandarización y demás constituyentes.

De este modo, a pesar de ser una propuesta adelantada en la década precedente, la IR ha concentrado desde su incepción un enorme interés en sus desarrollos, gracias a los antecedentes en el terreno de otros informes narrativos sobre capital intelectual, activos intangibles, CSR y gobierno corporativo (Abhayawansa, 2014; Rowbottom \& Locke, 2016; Rivera-Arrubla et al., 2016). Lo que se extiende incluso, en materia de información financiera e indicadores clave de desempeño.

La red de actores que respalda el IIRC busca establecer un marco basado en principios (IIRC, 2013b), planteado desde la desafiante labor de redefinir el contexto de la información corporativa a nivel mundial. Instituciones como el IIRC, a cargo de procesos trasnacionales de estandarización, definen tanto su capacidad para movilizar alianzas y recursos, como las restricciones a su tarea, a partir de las pautas establecidas por actores de mayor envergadura o más antiguos con la suficiente autoridad y legitimidad, como para poner en orden un campo de estandarización (Stone, 2000; Brown et al., 2009; Richardson \& Eberlein, 2011; Rowbottom \& Locke, 2016), quienes forman, además, una coalición de intereses mutuos que deciden su contexto de acción (Rowbottom \& Locke, 2016; Humphrey et al., 2017).

Bajo este panorama, la IR puede ser catalogada como el nuevo paradigma de los informes corporativos (Simnett \& Huggins, 2015) por lo que, de manera similar a lo que sucede con un paradigma de tipo científico, 
el éxito de este nuevo método radicará en atraer una masa crítica de adopciones (legitimidad), que le permitan ser auto-sostenible hasta que un competidor más fuerte amenace su supervivencia. Mientras esté en vigor dicho paradigma será tanto una base estable para la presentación de informes, como un elemento dado por sentado para la práctica profesional (Cooper \& Robson, 2006; Rowbottom \& Locke, 2016).

\section{La influencia de los organismos no estatales en la definición del IIRF}

Según Suchman (1995), la legitimidad puede portar un carácter pragmático, moral o cognitivo. La legitimidad pragmática descansa en los cálculos interesados de sus audiencias más inmediatas. Aquellas con las que la entidad entabla relaciones directas de intercambio, así como amplias interdependencias de tipo político, económico o social, que, en todo caso, implican consecuencias visibles para su propio bienestar, por lo que dichas audiencias estarían interesadas en hacer parte activa entre sus constituyentes, con el ánimo de escrutar sus comportamientos y determinar las consecuencias prácticas de sus actos sobre cualquiera de sus líneas de acción. Dado el respaldo reducido que este tipo de legitimidad contrae, un organismo estandarizador suele recurrir a otras fuentes. Por su parte, la legitimidad moral refleja una positiva valoración normativa que reside en el sistema de valores socialmente construido por una amplia audiencia interesada en el bienestar social. Esto la harían en principio menos manipulable que aquella que recoge exclusivamente consideraciones pragmáticas. Finalmente, la legitimidad cognitiva se basa en un tipo de conocimiento portador de credibilidad y/o garantía, que por una asunción cultural legitima al organismo y sus actividades como necesarios o inevitables.

Siguiendo a Giddens (1984), la efectividad de la influencia de cada agente depende de su posición estratégica. En este contexto, dicha efectividad consistiría en conseguir afectar la estructura de un estándar (o su contenido) o al organismo estandarizador. En este caso particular, los organismos de regulación que respaldan al IIRC participan en distintos frentes desde su fundación: forman parte, por ejemplo, de sus órganos de gobierno (IIRC, 2012a) y de las fuerzas de trabajo que redactaron el borrador del marco, y representan buena parte de sus fuentes de financiación. Además, participaron durante las dos consultas para posicionar su perspectiva, actuando como grupos de presión y formaron parte del selecto grupo a cargo de la definición del contenido publicado bajo la primera versión del IIRF. Es así que puede afirmarse que la legitimidad que este tipo de agentes aporta está fundada en una legitimidad pragmática, reducida a una suerte de legitimidad de intercambio consistente en respaldar una política o institución, a la expectativa de los beneficios particulares que un grupo de interés en particular pueda derivar de las mismas.

De otra parte, la literatura académica ha contrastado que los constituyentes que deciden participar activamente en un proceso público de emisión de estándares tienen la intención, no sólo de participar, sino de afectar sus resultados (Tutticci, Dunstan \& Holmes, 1994; Georgiou, 2005; Giner \& Arce, 2012). En esta línea, Georgiou (2004) afirma que la intención de influir sobre la producción de un marco o estándar puede ejercerse mediante diversos métodos y en distintas etapas del proceso. No obstante, destaca el envío de cartas de comentarios como un método oficial de lobbying, por el cual, el remitente hace pública sus respuestas, buscando que sus opiniones se materialicen en cambios en el estándar.

Precisamente, el organismo a cargo de lanzar un "debido proceso" cultiva la idea entre sus constituyentes que sus opiniones ejercerán algún grado de influencia sobre el contenido final de los estándares (Fogarty, 1994). Esto, dado que tanto participantes como observadores suponen que la legitimidad se sustenta en la audiencia colectiva como un todo, estando por tanto a salvo de las reservas de observadores individuales. En este contexto, el marco vigente es el resultado de una amplia convocatoria elevada a individuos, instituciones y organizaciones de todo el mundo, a partir de una política de debido proceso (IIRC, 2012b; 2013a) que comenzó con la consulta pública de un primer documento de debate en 2011 (IIRC, 2011) y que concluyó con la invitación a comentar sobre el borrador entre abril y julio de 2013 (IIRC, 2013a). En esta tarea, el IIRC 
recurrió a una estrategia de legitimidad moral (Suchman, 1995), convocando a distintos sectores como la empresa, los proveedores de capital, la profesión contable, la academia, la sociedad civil y la regulación (IIRC, 2012a, 2013a), actores con un dominio común sobre el campo de la información corporativa que validarían su trabajo y posición frente a la sociedad.

Dado que recurrir a una forma de legitimidad moral como lo es una consulta pública, supone en principio un procedimiento aséptico, neutral y democrático, analizamos cómo, no obstante, la posición estratégica de los organismos estudiados consiguió que este grupo de interés, plasmase sus puntos de vista en el contenido y orientación del marco, usando adicionalmente una suerte de legitimidad cognitiva asentada en la institucionalización del papel de este tipo de organismos como garantes del orden y deber ser. El análisis hecho pretende identificar cómo estos 'aliados clave' para el proceso de estandarización consiguieron influenciar varios aspectos fundamentales para la IR, imponiendo su visión sobre otros grupos (Jupe, 2000).

La fundamentación teórica y metodológica que guía esta indagación plantea que los agentes sociales, al tiempo que son influenciados o incluso determinados por estructuras, tienen la capacidad de influir en la sociedad mediante el uso de las reglas y recursos a su disposición (Giddens, 1984). A partir de esta perspectiva, estudiamos el contenido de las respuestas presentadas por agentes de estandarización no estatal, quienes participaron en la consulta de 2013, analizando principalmente la influencia de AICPA, GRI, SASB e IFAC, como actores estratégicos, quienes, gracias a su posición privilegiada, y a su conocimiento y experiencia en estos procesos, tuvieron mayores posibilidades de influir sobre la definición del marco. Para destacar el carácter de esta influencia, a continuación se identifica el impacto de sus comentarios, señalando cómo su posición fue acogida de manera especial, al momento de definir los principios y contenidos estudiados, y, en consecuencia, los posibles estándares que a futuro puedan emitirse a partir del IIRF.

\section{Diseño metodológico}

En esta investigación utilizamos una metodología de tipo narrativo-descriptivo, que consiste en estudiar, tanto la literatura académica como algunos documentos emitidos por el IIRC, que constituyen una base fundamental para el trabajo.

Para centrar el análisis, aquí se estudia la participación de aquellos organismos no estatales definidos en el segundo apartado y participantes de la consulta pública del marco. Luego de clasificar las 359 cartas recibidas por el IIRC en 2013, encontramos que 88 de estas fueron emitidas por estos organismos, 38 de las cuales, corresponden a cuerpos o asociaciones de carácter contable. Como procedimiento, en primer lugar, se revisa el 'IIRC due process' (IIRC, 2012b) que contiene las etapas y pautas para el proceso de debate y posterior formulación del marco. Además, se analiza el borrador del IIRF (IIRC, 2013a) y las cartas de comentarios enviadas por los agentes estudiados, disponibles en la página web del IIRC (2013e), revisando, además, el contenido de la primera versión del marco (IIRC, 2013b). Asimismo, se analizan los 'Technical agenda papers' (2013f), documentos en los que el Equipo de Trabajo y el Consejo del IIRC, sintetizan los resultados del proceso, y que sirvieron como base de apoyo durante la redacción definitiva del marco. Finalmente, para ampliar el contexto de análisis se revisan el documento de Gobierno (IIRC, 2012a) y la Estrategia de Futuro del IIRC (2013d), en los que se definen su estructura y propósitos.

En distintas fases de esta investigación recurrimos al análisis de contenido para explorar las fuentes estudiadas (Berg, 2004). Identificamos las principales fases de evolución del IIRC y el IIRF, así como los principales actores involucrados. En este caso, de manera similar a Flasher, Luchs, \& Souza (2018), quienes analizan el papel de la profesión contable en el establecimiento de estándares de sostenibilidad, recurrimos a un análisis cualitativo para analizar el papel estratégico de los cuerpos de regulación privada sobre el IIRF. Así, en lugar de adoptar una metodología cuantitativa para medir las respuestas de los participantes, a partir de clasificaciones que generalmente las ubican como "a favor" o "en contra" (e.g., Deegan, Morris, \& 
Stokes, 1990; Reuter \& Messner, 2015), o aquellas que intentan codificar algunos matices sobre los reclamos por cambios menores, moderados o importantes en el estándar (Bamber \& McKeeing, 2016), esta vez, se interpretan las cartas recibidas por el IIRC como portadoras de significado, a partir de un enfoque más profundo que permita reconocer la fuerza de cada posición declarada y su nivel de influencia. En este sentido, el propósito es analizar el significado manifiesto y luego el contenido latente (Berg, 2004) de las cartas de comentarios estudiadas, a partir de la lectura en profundidad de cada documento, y posteriormente, de un estudio que permita identificar las categorías de análisis alrededor de la definición de la IR y la demarcación de su público de interés. Hecho esto, se realiza un análisis temático para identificar, analizar y reconocer patrones (temas) en los contenidos (Braun \& Clarke, 2006) relacionados con las categorías objeto de estudio (Fereday \& Muir-Cochrance, 2006). Finalmente, se contrastan: (1) el borrador de consulta, (2) las cartas de los agentes estudiados, y (3) el marco IIRF, estableciendo si existe o no una directa correspondencia entre los cambios observados y los comentarios de estos organismos.

En la última fase del estudio, y una vez los resultados preliminares señalaron que si bien, en términos generales, las cartas de los organismos de estandarización privada, como agentes clave en asegurar la legitimidad cognitiva (Suchman, 1995) del marco, sugieren aspectos relevantes a ser considerados, de manera particular, fueron las cartas firmadas por AICPA, GRI, SASB, e IFAC, las que dada la influencia (posición estratégica) de estos agentes frente al IIRC, consiguieron un impacto visible y directo (legitimidad pragmática) en el contenido definitivo publicado como la primera versión oficial. Motivo por el cual, los resultados que se presentan a continuación, están dedicados a señalar y discutir los principales aspectos que constatan este hallazgo.

\section{Hallazgos: cambios en el IIRF a partir de las cartas enviadas por organismos no estatales}

En este apartado exponemos los principales resultados del trabajo, analizando aquellas observaciones y comentarios hechos por los organismos que constituyen nuestra unidad de análisis, y que consiguieron influir sobre el contenido del marco. Producto del análisis temático definido en el diseño metodológico, estos hallazgos se estructuran asociando su contenido a los aspectos definidos inicialmente como objeto de estudio. A continuación se analiza la incidencia de estos actores sobre: (1) la definición de un marco basado en principios; (2) el concepto de Informe Integrado como informe independiente, y la Información Integrada como proceso; y (3) la demarcación del público destinatario del informe.

\section{El IIRF: un marco basado en principios}

El enfoque basado en principios (principles-based) permite la aplicación de normas a países con diversas tradiciones y condiciones institucionales, gracias a su flexibilidad. Además, se considera que un sistema basado en principios hace referencia a interpretaciones básicas que por su jerarquía superior pueden dominar cualquier otra regla establecida (Carmona \& Trombetta, 2008). Así pues, los sistemas basados en principios establecen normas genéricas convenientes al propósito de entregar un marco general, a partir del cual puedan desprenderse reglas específicas a futuro. A diferencia de los sistemas basados en reglas, un marco basado en principios no aborda todos los temas en detalle. En su lugar, define conceptos con cierta amplitud, a la vez que ambigüedad, de modo que los estándares que se desprendan a partir de éste, puedan regirse bajo su influencia.

Como veíamos en el segundo apartado, las organizaciones tienen cierta preferencia por demarcar sus propias reglas (auto-regulación), y ajustarlas luego, a la conveniencia de sus intereses y recursos (soft law). Esta práctica convergente con una tipología de estándares basados en principios hace deseable el someterse a las buenas prácticas ampliamente respaldadas por la sociedad (legitimidad moral). Esto, al tiempo que se 
exhiben públicamente los esfuerzos realizados por observar un comportamiento ético y responsable, a través, por ejemplo, de la entrega voluntaria de informes corporativos.

En primer lugar, y ya que un planteamiento fundamental del IIRF es su definición como marco 'basado en principios', en lugar de 'basado en reglas' (IIRC, 2013a, 2013b, Cheng, Green, Conradie, et al., 2014), este es un elemento clave a la hora de establecer la relevancia de los cambios introducidos al marco durante la consulta. Aunque la preferencia por este posicionamiento ya aparecía, por ejemplo, desde el Informe Trueblood emitido por el AICPA en 1973 (Zeff, 1999; Damant, 2003), su consideración en el IIRF refleja una tendencia guiada por demandas internacionales, así como la dependencia de unas audiencias inmediatas (legitimidad pragmática), que abogan por el establecimiento de estándares lo menos prescriptivos posibles y administrados en un ámbito de regulación privada.

Frente a este tema, instituciones como el AICPA (ref. 1201), Climate Disclosure Standards Board (CDSB) (ref. 193) e IFAC (ref. 219), en sus cartas de comentarios utilizan recursos atribuibles a una legitimidad moral que harían incontestables sus requerimientos al prevenir acerca de los efectos nocivos de utilizar un lenguaje prescriptivo y contrario al espíritu de un marco basado en principios, por lo que sugieren evitar términos como 'cumplir' y 'requisitos' y emplear, en su lugar, otros que indiquen la necesidad de 'aplicar' los principios correctamente. El AICPA también apoya un marco de indicadores no específicos o métodos de medición, sugiriendo que sea el propio mercado quien los desarrolle, argumentando que deben ser las organizaciones, las llamadas a determinar el mejor enfoque para la presentación de informes, sin que haya de por medio una temprana intervención reguladora que frene la innovación.

Por su parte, el Integrated Reporting Committee (IRC) de Sudáfrica (ref.189), apoya un enfoque basado en principios que parta del enfoque de 'aplicar o explicar'. Dada la experiencia del IRC, organismo que lidera la adopción obligatoria de la IR en las compañías cotizadas, resulta de especial relevancia su postura en defensa de establecer un equilibrio entre proporcionar orientación a los preparadores y permitirles flexibilidad para la innovación.

SASB (ref. 048) se une al grupo de organismos que avalan un 'principles-based approach', afirmando que este proyecto complementa su propio enfoque, siendo además, compatible con las normas de divulgación pública que rigen los mercados de capitales de los Estados Unidos.

Estas consideraciones fueron recogidas en los apartados 1.17 y 1.18 del marco, en los que se define al IIRF como un marco basado en principios. La tabla 1 señala las posiciones defendidas en las respuestas analizadas, presentando luego, la versión original del borrador, y finalmente, la definición de este aspecto en la versión definitiva.

En términos generales, los cambios en la redacción final del marco señalan un paso desde la demanda inicial de aplicar todos los requisitos señalados sin excepción, a la solicitud de aplicar todos aquellos requisitos disponibles, siempre y cuando esto no cause daño a la compañía, ni se pasen por alto las prohibiciones legales. Este ajuste, representa una mayor flexibilidad para el cumplimiento del IIRF, aun tratándose de un marco basado en principios.

Además, la primera versión oficial del marco ya no habla de identificar aquella información que haya sido omitida, sino que, en su lugar, se limita a solicitar que se indique la naturaleza de la misma. En consecuencia, además de valerse de la flexibilidad de este enfoque, el carácter auto-regulatorio de los estándares producidos por organismos no estales, ofrece la garantía de dar cierto margen del que no sólo toman ventaja la experimentación e innovación, como se sugiere, sino la facilidad de adaptación a los medios disponibles para 'aplicar y explicar' ciertos patrones de divulgación. Esto, mientras se omite información que bien puede ser material para los stakeholders y la sociedad, lo que adicionalmente, debilita el propósito inicial porque la IR estuviese pensada para narrar una historia de creación de valor en el tiempo, limitando una rendición de cuentas verdaderamente transparente e integral. 
TABLA 1

La influencia de organismos privados sobre la definición de un IIRF basado en principios

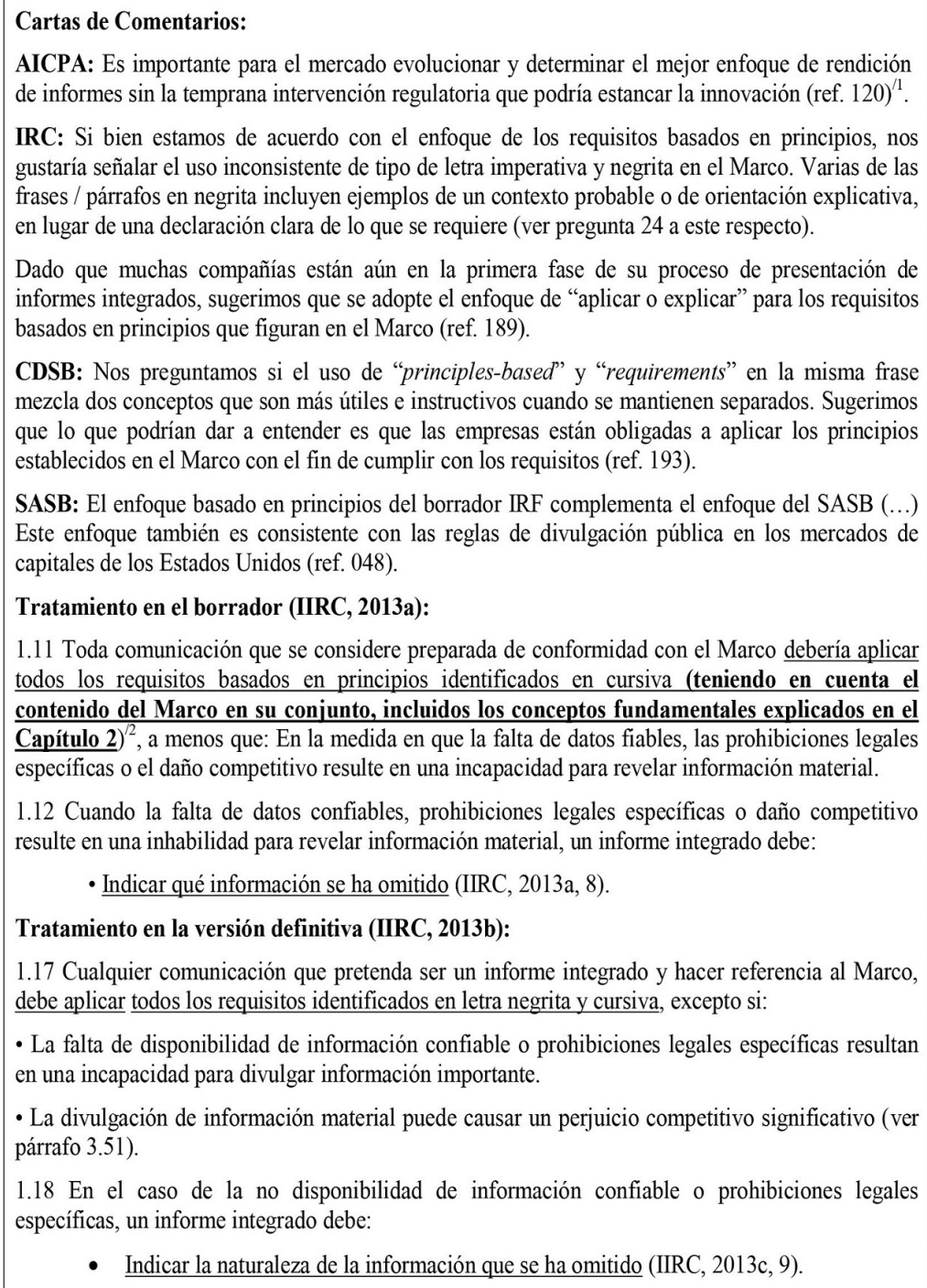

CDSB: Nos preguntamos si el uso de "principles-based" y "requirements" en la misma frase mezcla dos conceptos que son más útiles e instructivos cuando se mantienen separados. Sugerimos que lo que podrían dar a entender es que las empresas están obligadas a aplicar los principios establecidos en el Marco con el fin de cumplir con los requisitos (ref. 193).

SASB: El enfoque basado en principios del borrador IRF complementa el enfoque del SASB (...) Este enfoque también es consistente con las reglas de divulgación pública en los mercados de capitales de los Estados Unidos (ref. 048).

Tratamiento en el borrador (IIRC, 2013a):

1.11 Toda comunicación que se considere preparada de conformidad con el Marco debería aplicar todos los requisitos basados en principios identificados en cursiva (teniendo en cuenta el contenido del Marco en su conjunto, incluidos los conceptos fundamentales explicados en el $\underline{\text { Capítulo 2 }}^{2}$, a menos que: En la medida en que la falta de datos fiables, las prohibiciones legales específicas o el daño competitivo resulte en una incapacidad para revelar información material.

1.12 Cuando la falta de datos confiables, prohibiciones legales específicas o daño competitivo resulte en una inhabilidad para revelar información material, un informe integrado debe:

- Indicar qué información se ha omitido (IIRC, 2013a, 8).

Tratamiento en la versión definitiva (IIRC, 2013b):

1.17 Cualquier comunicación que pretenda ser un informe integrado y hacer referencia al Marco, debe aplicar todos los requisitos identificados en letra negrita y cursiva, excepto si:

- La falta de disponibilidad de información confiable o prohibiciones legales específicas resultan en una incapacidad para divulgar información importante.

- La divulgación de información material puede causar un perjuicio competitivo significativo (ver párrafo 3.51 ).

1.18 En el caso de la no disponibilidad de información confiable o prohibiciones legales específicas, un informe integrado debe:

- Indicar la naturaleza de la información que se ha omitido (IIRC, 2013c, 9).

1/ Las cartas originales fueron escritas en inglés, por lo que los fragmentos citados en español corresponden a una traducción libre del autor.

2/ Los subrayados son del autor. Su propósito es destacar los puntos analizados. El texto en negrita pretende, además, resaltar los extractos eliminados de la versión final del marco.

Fuente: elaboración propia.

\section{El Informe Integrado como informe independiente}

En cuanto a su definición y presentación, el borrador definía el IR como el informe principal de las organizaciones. No obstante, la declaración que afirma que este sería un informe independiente (IIRC, 2011; 2013a), que transformaría las estructuras vigentes sobre presentación de informes, no se mantuvo en el marco publicado, por lo que en los apartados 1.12 a 1.16, relativos a este aspecto, encontramos un cambio de orientación que mantiene dichas estructuras como habían sido planteadas en el pasado. Dicho cambio, se vale de una estrategia de legitimidad cognitiva, que retira la concepción del IR como informe único o autónomo 
(apartado 1.18 del borrador, IIRC 2013a), regresando el control a los actores, que se supone, deben velar por su cuidado sin desafiar las formas dadas por sentadas.

$\mathrm{Al}$ revisar qué respuestas pudieron influir directamente sobre el concepto de IR como único informe, encontramos cómo el AICPA (ref. 120) señala que a algunas empresas les podría resultar más conveniente aplicar el proceso IR a canales de información corporativa ya establecidos; es decir, incorporar los elementos del marco en sus estructuras de reporte; tarea distinta a la de elaborar un IR independiente. A esta misma preocupación, sobre la presentación del informe se sumó la manifestada en la carta del IFAC (ref. 219). En respuesta a estas inquietudes, el marco cambió la definición del IR como informe independiente, indicando en el punto 1.15, que un IR puede ser bien, un informe independiente, o incluirse como parte "distinguible, prominente y accesible" (IIRC, 2013b, p. 12) de otro informe o comunicación. Los apartados consignados en la tabla 2, permiten visualizar el hallazgo señalado.

TABLA 2

La influencia de organismos privados sobre la definición del IR como informe independiente

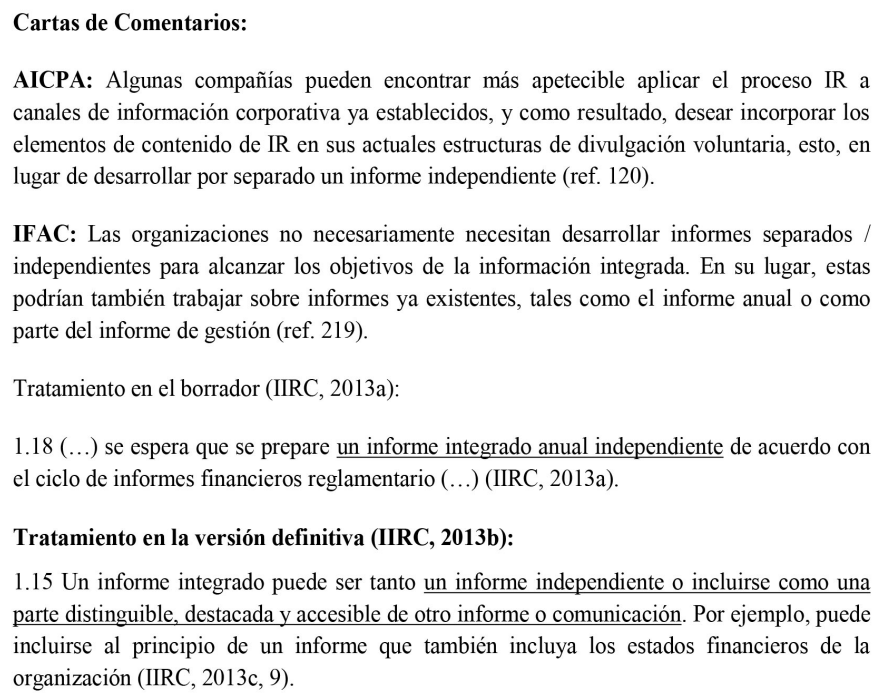

Fuente: elaboración propia.

Respecto de si hablar de IR significa hablar de 'One Report', Tweedie y Martinov (2015) consideran que ya que el libro del mismo título, de autoría de Eccles y Krzus (2010), antecede la existencia del IIRC, y dado que el IR se ha desarrollado a través de su propio proceso de discusión y consulta no puede suponerse claramente que dicho término pueda representar, ni el concepto de IR del IIRC, ni la agenda de este organismo. No obstante, el concepto de IR había estado hasta entonces estrechamente relacionado con la imagen de un informe único e independiente (Eccles \& Krzus, 2010; Simnett \& Huggins 2015; Rowbottom \& Locke, 2016), incluso antes de la introducción del IIRC (Kolk, 2003, 2010). En consecuencia, si bien el concepto IR puede suponer en principio la imagen de un informe único y conectado, es importante explorar hasta qué punto, éste debería o no, ser sinónimo de presentar todo el contenido (económico, social, medioambiental y de gobierno corporativo) de las organizaciones bajo un único documento.

De otra parte, algunos autores consideran que el IR no debería limitarse a ser sólo una yuxtaposición de distintas categorías que antes permanecían aisladas, sino más bien, debería significar el paso hacia una narrativa consolidada del desempeño corporativo (Jensen \& Berg, 2012; Zicari, 2014), que exprese la conexión interna entre las unidades funcionales del negocio y la cadena de valor en conjunto. En consecuencia, lo que el concepto de IR debe transmitir no es necesariamente la producción de un único informe sino, más bien, la concepción que toda la información publicada deba ser producida como resultado de un proceso que permita una clara alineación y conectividad, a través de referencias a los distintos componentes del informe, o a informes adicionales más detallados (Hindley \& Buys, 2012). Flower (2015) y Thomson (2015) llaman precisamente la atención sobre las escasas posibilidades del IR como informe único, invitando a revisar si este aspecto significa una garantía de integridad y transparencia. 


\section{El Informe Integrado como proceso}

El borrador del marco concebía a la IR como proceso, y no sólo como una comunicación o resultado. No obstante, esta visión desaparece, y al parecer, la carta dirigida por IFAC (ref. 219), tuvo gran ascendencia sobre este cambio en particular. A este respecto, dicha carta critica de manera directa el que se califique a la IR como proceso. Esto, al menos, mientras no se haya definido claramente qué significa la expresión: el proceso del marco integrado, y, en consecuencia, qué es exactamente aquello que debe ser aplicado continuamente a todos los informes y comunicaciones. Parece entonces, que a este comentario debemos el que la visión de la IR como proceso no haya sido mantenida en la versión oficial. Este hecho se constata en la eliminación del contenido de los puntos 1.2 y 1.18 .

IFAC, además, consiguió un impacto adicional respecto de los objetivos del marco, y el mismo está directamente vinculado a su intención de cuestionar el enfoque de la IR como proceso. Su carta de comentarios sugirió enmendar el objetivo del IIRF, cambiando el texto del párrafo 1.9, al considerar que su propósito primario debería ser ayudar a las organizaciones a determinar el contenido de sus informes, antes que a desarrollar su proceso IR. En consideración, el apartado 1.3 ahora señala como propósito, el establecer los principios rectores y elementos que rigen el contenido global de un IR, y explicar los conceptos fundamentales que lo sustentan. Veamos estos cambios en la tabla 3.

TABLA 3

La influencia de organismos privados sobre el concepto del IR como proceso

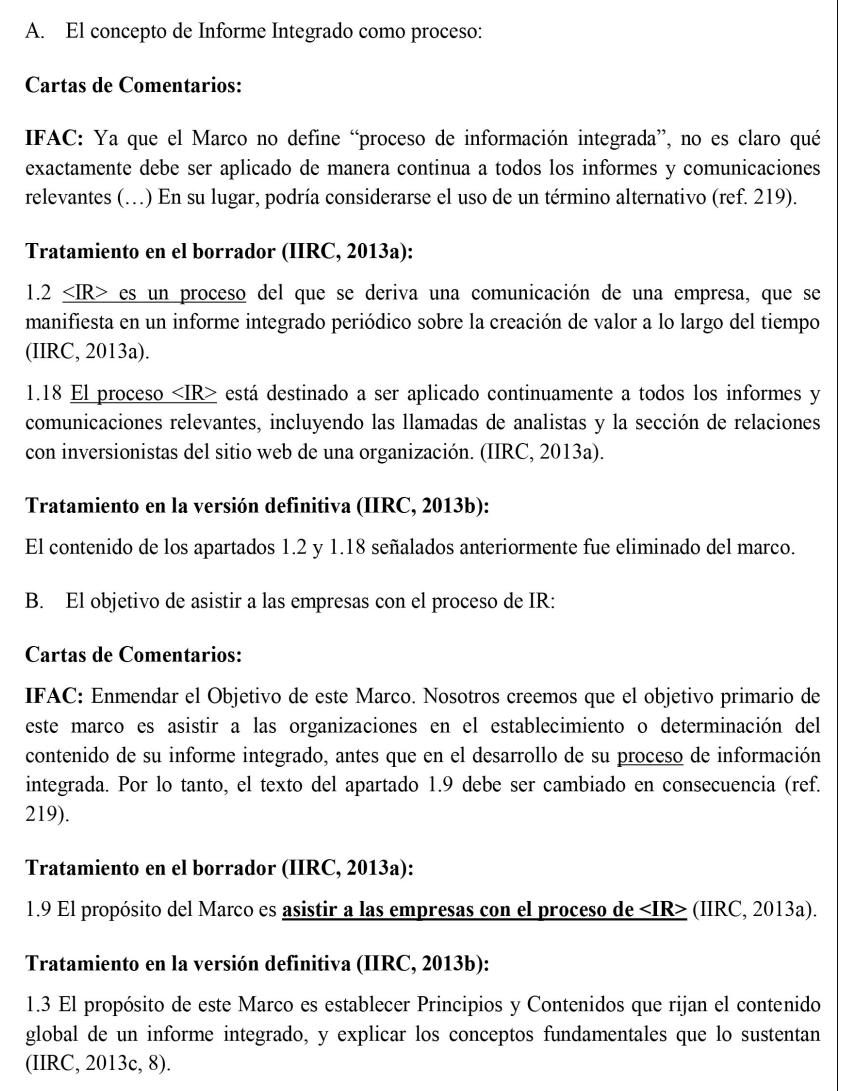

1/ La palabra "proceso" aparece subrayada en la carta enviada por el IFAC.

Fuente: elaboración propia.

Como resultado, y aunque en el Glosario del marco (IIRC, 2013b), el término Integrated Reporting se define como proceso, a partir de una estrategia de legitimidad cognitiva que invoca el mantenimiento de las estructuras ya dadas por sentadas para evitar la confusión que podría causar el vincular el concepto de informe al de proceso, las demás referencias vinculadas con esta idea, fueron eliminadas, manteniendo sólo algunas alusiones marginales al proceso de determinación de asuntos materiales y al proceso de creación de valor. 


\section{El público destinatario de la Información Integrada}

Finalmente, analizamos la definición del público destinatario del informe, encontrando que IFAC considera oportuno que cada organización determine cuáles deben ser los usuarios previstos; mientras AICPA apoya el contenido de los párrafos 1.6 a 1.8, que identifican a los proveedores de capital financiero como público principal. En consonancia con el AICPA, GRI señala estar de acuerdo con este enfoque, y recomienda, además, que el principio de Materialidad se centre en sus intereses, posición a la que se suma SASB (ref. 048), al opinar que al igual que ocurre con los SECfilings requeridos por the U.S. Securities and Exchange Commission (SEC) y presentados por las compañías que cotizan en bolsa en los Estados Unidos, es necesario que el IR esté destinado a beneficiar a los proveedores de capital. La tabla 4 señala los cambios en este aspecto.

TABLA 4

La influencia de organismos privados sobre la definición del público objetivo del informe

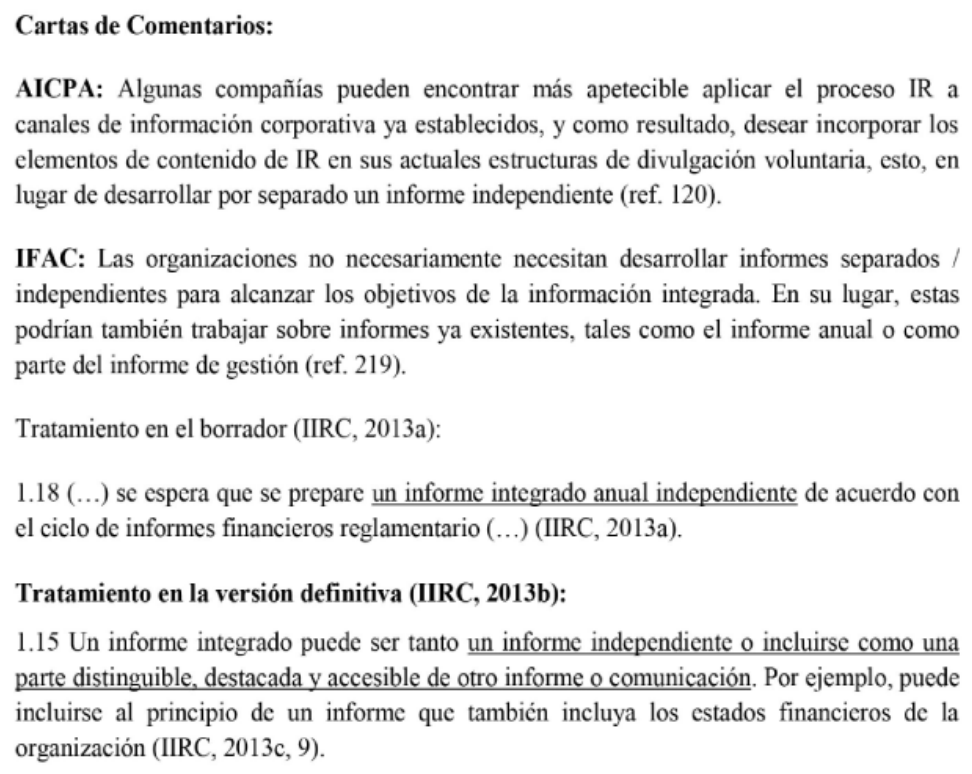

$1.15 \mathrm{Un}$ informe integrado puede ser tanto un informe independiente o incluirse como una parte distinguible, destacada y accesible de otro informe o comunicación. Por ejemplo, puede incluirse al principio de un informe que también incluya los estados financieros de la organización (IIRC, 2013c, 9).

Fuente: elaboración propia.

Aunque los argumentos esbozados suelen ser presentados bajo un cuidadoso manejo del lenguaje, es posible advertir cómo de manera más o menos sutil, los intereses de un grupo particular (legitimidad pragmática), son presentados en el marco de una consulta pública validada por una estrategia de debido proceso, y por tanto, calificable en principio bajo la dimensión de una legitimidad moral. Como puede observarse, finalizada la consulta se refuerza que un IR debe dirigirse principalmente a los proveedores de capital, dejando claro que los demás grupos de interés serán beneficiarios de la información, siempre que estos se interesen en la habilidad de la organización para crear valor en el tiempo (IIRC, 2013b). Este asunto ha recibido fuertes críticas (e.g. Cheng et al., 2014, Flower, 2015; Tweedie \& Martinov, 2015) ya que, por ejemplo, el IIRC no expone con argumentos de peso cómo los intereses de los inversores podrían llegar a alinearse en el largo plazo con los de otras partes interesadas (Tweedie \& Martinov, 2015).

\section{Discusión de los resultados}

El cambio en los aspectos clave del marco que aquí se identifican, y su conexión con las cartas enviadas por los organismos analizados, dan cuenta de que a pesar de tratarse de un proceso abierto a consulta pública, algunos grupos o agentes ostentan una posición aventajada frente al proceso de producción de un estándar, ventajas que se derivan del lugar ocupado por los participantes en sus propios contextos sociales (posición estratégica). Las acciones de estos grupos, tienen vastos efectos, tanto en tiempo como en espacio (Giddens, 
1984), afectando distintas sendas espacio-temporales gracias a su poder económico, social, cultural, simbólico y político.

Los resultados de este trabajo permiten afirmar que pese a partir de un llamado abierto a diversos grupos (legitimidad moral), los intereses de las audiencias inmediatas (legitimidad pragmática) fue predominante en el proceso, instrumentalizando algunas formas proveedoras de credibilidad y/o garantía (legitimidad cognitiva). En este caso, se observa de manera predominante, el poder de la legitimidad pragmática expresada a través de una legitimidad de intercambio, consistente a su vez, en un sub-tipo de legitimidad más social: la legitimidad de influencia (Suchman, 1995). Mediante esta última, un constituyente en particular (los organismos privados), respalda al IIRC, no solamente a cambio de la provisión de un intercambio específico a su favor, sino de atener los intereses más amplios del grupo al que se suscribe (los proveedores de capital).

Así, de acuerdo con su visión e intereses, cada participante tomará la decisión de adoptar un determinado conjunto de estrategias para incidir en el marco, según pretenda, por ejemplo, cambiar, destacar o reprimir un aspecto específico (Tutticci et al., 1994; Giner \& Arce, 2012; Reuter \& Messner, 2015). Más, tratándose de agentes ubicados en el corazón de los grupos de trabajo y en las mesas de decisión del organismo consultante, donde la participación de profesionales y reguladores suele presentarse en términos de una legitimidad cognitiva (Suchman, 1995); esto es, por su interés en legitimar su papel como expertos y su misión de contribuir al buen funcionamiento de sus campos de especialización (Oprisor, 2014), mientras consiguen garantizar que su visión o perspectiva prevalezca.

En línea con estos hallazgos, Jupe (2000) concluye que la legitimidad de un standard-setter está atada a la aprobación proveniente de un pequeño grupo de participantes en la consulta, considerados como 'aliados clave'. Su investigación revela cómo durante el proceso de emisión del Financial Reporting Standard No. 1, el Accounting Standards Board (ASB) se vio obligado a dar un paso atrás en su posición inicial sobre aspectos importantes, para mantener el apoyo de sus aliados y conseguir la adopción del mismo. En este caso, las firmas de auditoría, los organismos profesionales y los preparadores, recurrieron a una retórica de autoreferenciación en sus cartas para persuadir al organismo estandarizador de enmendar este estándar de acuerdo con sus propios objetivos.

En consecuencia, los resultados de este análisis permiten constatar que tanto la evolución, como la fase actual de desarrollo de la IR, dependen de manera importante de la red de aliados erigida a su alrededor; mientras sus principios hoy son un asunto de interés que reúne a importantes instituciones en el terreno de normalización de estándares corporativos. En este escenario, el IIRC trabaja actualmente en cerrar la brecha hacia la adopción generalizada de la IR como la norma de presentación de informes, por lo que a partir de 2012 ha suscrito una serie de Memorandos de Entendimiento (MoU) con otros cuerpos de estandarización, entre los que las instituciones analizadas en esta investigación ocupan un lugar destacado. La alineación reclamada por estos organismos podría ser consecuencia de que hasta ahora, grandes recursos y esfuerzos hayan estado dispersos alrededor de un objetivo cercano: la estandarización de prácticas de presentación de informes. Por lo que más allá de asegurar que la IR se presente como la solución ideal o el paso natural hacia el futuro en la rendición de cuentas, este movimiento podría corresponder a una estrategia que busque perpetuar las relaciones de poder pre-existentes y concentrar más eficientemente los recursos, consiguiendo así y con mayor eficacia, el atraer la adopción global y un uso más amplio que el alcanzado antes por otras directrices.

Bajo esta estrategia, el IIRC lanzó el Corporate Reporting Dialogue $(\mathrm{CRD})^{2}$ en la Conferencia Anual de la Red Internacional de Gobierno Corporativo (ICGN), celebrada en Ámsterdam en junio de 2014. Los organismos participantes de esta iniciativa: The Financial Accounting Standards Board (FASB), International Public Sector Accounting Standards Board (IPSASB), International Organization for Standardization (ISO), CDP, CDSB, GRI, IASB, SASB, y por supuesto, el propio IIRC, se plantean el propósito de desarrollar un "Paisaje de Información Corporativa" que ponga de relieve la conectividad de los diversos marcos y estándares de información y su importancia para la información integrada. CRD es sin duda, un nuevo paso en el camino de cerrar la brecha hacia la adopción generalizada del denominado One Report, asegurando el predominio 
de los proveedores de capital como su público destinatario. Su propósito general es alinear la dirección, contenido y desarrollo de los marcos liderados por las organizaciones participantes, promoviendo la creación de valor para los proveedores de capital financiero.

Finalmente, en la tarea de asegurar la acogida global de la IR, durante el año 2020, el IIRC abrió una nueva convocatoria de 90 días (IIRC, 2020) para que individuos, corporaciones y agencias de todo el mundo se vinculen a un nuevo proceso público de revisión del marco de referencia, con el objetivo de lanzar la segunda versión del IIRF a finales de este mismo año. Este evento hace pertinente poner en cuestión distintos aspectos alrededor de la formulación y/o revisión de los principios de la IR, dadas sus posibles consecuencias, no sólo sobre las corporaciones y la academia, sino también sobre el diseño de políticas públicas que habrán de afectar a comunidades ubicadas en distintos contextos geográficos, en principio, ajenos a las lógicas e intereses que subyacen a su producción.

\section{Conclusiones y reflexiones}

La información corporativa es tema de interés general, no sólo para los proveedores de capital y administradores, sino para un grupo cada vez más amplio de partes interesadas, por lo que puede decirse que la divulgación de información empresarial es también un asunto de política internacional. Este hecho ha propiciado que algunas instituciones y agentes privados vengan ganando un importante terreno en la tarea de sugerir, definir y supervisar la generación de estándares financieros y no financieros, que hoy resultan esenciales en la evolución de la IR.

Como resultado, la definición de principios para la preparación y presentación de la información es liderada actualmente por organismos de naturaleza privada. Entre ellos, destacamos el impacto de AICPA y FASB, ambos con una larga tradición en proyectos sobre información corporativa, GRI y sus ampliamente conocidos estándares de sostenibilidad, y IASB, que con sus estándares internacionales de información financiera, lidera un proceso de armonización internacional, impensable apenas hace algunos años.

En esta misma senda, y a pesar de ser un fenómeno relativamente reciente y objeto de amplia contestación (Paternostro, 2020), la IR viene ganando un fuerte impulso. En el pasado, la información aislada sobre capital intelectual, CSR, gobierno, finanzas, y otras declaraciones específicas, estaban en el centro de atención; no obstante, en la actualidad, la mirada de diversas partes interesadas se concentra en los informes integrados.

El IIRC es resultado de experiencias anteriores en materia de formulación de políticas no estatales; mientras el IIRF corresponde a la evolución de marcos previos diseñados principalmente sobre bases no obligatorias planteadas desde las dimensiones financiera, social y ambiental (Rowbottom \& Locke, 2016; Abhayawansa, 2014). Podría decirse, que la IR es una tendencia relativamente nueva, que, no obstante, bebe de fuentes de vieja data. Esto hace que su marco sea receptor de desarrollos e intereses que persiguen un impacto más duradero y de mayor alcance. En este sentido, un proceso de institucionalización como la formación del IIRC estaría profundamente afectado por las estrategias de legitimación planteadas por sus fundadores, así como por los intereses que estos tengan en que se reproduzcan las relaciones de poder pre-existentes (Brown et al., 2009; Rowbottom \& Locke, 2016). De otro modo, estaríamos ante un proceso de desarrollo menos intensivo y con menores posibilidades de "imponer" un modelo de presentación de informes corporativos de dimensiones globales.

A pesar de señalarse como un debido proceso planteado en términos de una legitimidad moral, que como tal, ofrece a las partes interesadas múltiples oportunidades para expresar su posición, asegurar la apertura de sus procedimientos y facilitar la cuidadosa consideración de todas las posiciones (Fogarty, 1994), en términos que aportan garantía y credibilidad sobre los resultados (legitimidad cognitiva), el análisis realizado, adelantado desde una perspectiva política sobre los efectos de la regulación privada en procesos de estandarización internacional, constata una validación del IIRC apuntalada en formas de legitimidad 
pragmática. Esto, a partir del impacto de un reducido grupo de organismos no estatales como GRI, AICPA, IFAC y SASB en su formación, y en particular, en el desarrollo de los principios rectores del IIRF. Lo que tendrá efectos no sólo sobre iniciativas privadas a nivel corporativo, sino posiblemente sobre la definición de políticas públicas y sus consecuencias en distintas regiones del globo. Los cambios conseguidos por las comunicaciones estudiadas, permiten vislumbrar el papel decisivo de estos participantes en el contenido y estructura del marco. Principalmente, cabe destacar, cómo estos influyeron sobre la decisión de puntos esenciales relativos a la definición del IR, la orientación del marco y el público objetivo. Y cómo, el movimiento hacia la adopción general de estos informes continúa abriéndose camino a partir de suscribir memorandos y alianzas entre organismos trasnacionales de estandarización. Estrategias que lejos de desafiar o contestar los dominios de instituciones precedentes como el GRI, buscan construir una red estable y sólida alrededor del apoyo a las iniciativas sobre IR.

En este sentido, el presente trabajo permite concluir, cómo a partir de la experiencia y recursos de otros organismos, el IIRC pretende asegurar su propia supervivencia y la de sus estándares de rendición de cuentas (Humphrey et al., 2017). Hecho, que se proyecta al parecer, más como una estrategia de manejo de impresión ocupada en cambiar los arquetipos de diseño, y que en tanto no representa una verdadera transformación en el ADN de las organizaciones (Paternostro, 2020).

Se espera que este trabajo pueda arrojar algunas luces sobre la comprensión de futuros procesos de consulta de estándares y principios de similar naturaleza. En particular, este artículo podría contribuir al análisis del proceso público de actualización del marco adelantado por el IIRC entre mayo y agosto de 2020.

Dadas las implicaciones actuales de los procesos de formulación de políticas, especialmente de aquellos de iniciativa privada, sobre distintos sectores de la vida, sin lugar a dudas, resulta fundamental que la academia continúe trabajando en analizar los procesos de consulta adelantados por instituciones como el IIRC, atendiendo especialmente a la evolución de su marco de referencia y/o estándares. Esto, tanto a partir de la contribución que pueda hacerse desde distintos análisis teóricos como del planteamiento de metodologías alternativas, así como de los hallazgos de trabajos empíricos que permitan estudiar, bajo distintas perspectivas, las verdaderas posibilidades de futuro de iniciativas como la IR, debatiendo además, sus posibles consecuencias para la profesión contable, las organizaciones, la sociedad, el medio ambiente y la economía global.

\section{Consideraciones éticas}

Informo que el artículo presentado no requiere del aval de un Comité de Ética o Bioética, dado que la investigación no utiliza ningún recurso vivo, agentes o muestras biológicas, datos personales, entrevistas, encuestas o resultados diagnósticos, ni requiere de procedimientos que representen riesgo de ninguna índole sobre la vida, el ambiente o los derechos humanos.

\section{Contribución de los autores}

El artículo presentado fue concebido y ejecutado completamente por la autora que firma el trabajo.

\section{Conflictos de interés}

La autora declara no tener ningún tipo de conflicto de interés asociado al desarrollo de la investigación o a la presentación del artículo. 


\section{Agradecimientos}

Este artículo fue realizado por la autora, como parte de su trabajo de investigación como profesora universitaria, bajo remuneración en calidad de docente de tiempo completo de la Universidad del Valle. No obstante, no corresponde a un proyecto de investigación financiado y fue elaborado en su mayoría (en un $80 \%)$ durante sus horas de descanso y vacaciones.

\section{Referencias}

Abeysekera, I. (2013). A template for integrated reporting. Journal of Intellectual Capital, 14(2), 227-245. https://d oi.org/10.1108/14691931311323869

Abhayawansa, S. (2014). Milestones in the Development of Intellectual Capital Reporting. International Journal of Business and Management, 9(2), 114-123. https://doi.org/10.5539/ijbm.v9n2p114

American Institute of Certified Public Accountants - AICPA (2014). Changing Landscape of Corporate Reporting. ht tps://competency.cgma.org/media_resources/206619-changing-landscape-of-corporate-reporting

Bamber, M. \& McMeeking, K. (2016). An examination of international accounting standard-setting due process and the implications for legitimacy. The British Accounting Review, 48(1), 59-73. https://doi.org/10.1016/j.bar.20 15.03.003

Bartley, T. (2005). Corporate accountability and the privatization of labor standards: Struggles over codes of conduct in the apparel industry. Research in Political Sociology, 14, 211-244. https://doi.org/10.1016/S0895.9935(05)1 JU07-8

Bartley, T. (2007). Institutional emergence in an era of globalization: The rise of transnational private regulation of labor and environmental conditions. American Journal of Sociology, 113(2), 297-351. https://doi.org/10.1086 1518871

Bebbington, J., Kirk, E. A., \& Larrinaga, C. (2012). The production of normativity: a comparison of reporting regimes in Spain and the UK. Accounting, Organizations and Society, 37(2), 78-94. https://doi.org/10.1016/j.aos.2012 .01 .001

Beisheim, M., \& Dingwerth, K. (2008). Procedural legitimacy and private transnational governance. Are the good ones doing better? SFB-Governance Working Paper Series, 14, 3-32. https://doi.org/10.17169/refubium-22858

Bendell, J., Miller, A., \& Wortmann, K. (2011). Public policies for scaling corporate responsibility standards. Sustainability Accounting. Management and Policy Journal, 2(2), 263-293. https://doi.org/10.1108/2040802 1111185411

Berg, B. L. (2004). Qualitative research methods for the social sciences. Boston, MA: Pearson.

Braun, V., \& Clarke, V. (2006). Using thematic analysis in psychology. Qualitative Research in Psychology, 3(2), 77-101. https://doi.org/10.1191/1478088706qp063oa

Brown, H. S., de Jong, M., \& Levy, D. L. (2009). Building institutions based on information disclosure: Lessons from GRI's sustainability reporting. Journal of Cleaner Production, 17(6), 571-580. https://doi.org/10.1016/j.jclepr o.2008.12.009

Camilleri, M. A. (2018). Theoretical insights on Integrated Reporting: The inclusion of non-financial capitals in corporate disclosures. Corporate Communications: An International Journal, 23(4), 567-581. https://doi.org/1 0.1108/CCIJ-01-2018-0016

Carmona, S., \& Trombetta, M. (2008). On the global acceptance of IAS/IFRS accounting standards: The logic and implications of the principles-based system. Journal of Accounting and Public Policy, 27(6), 455-461. https://d oi.org/10.1016/j.jaccpubpol.2008.09.003

Cashore, B. (2002). Legitimacy and the privatization of environmental governance: How non-state market-driven (NSMD) governance systems gain rule-making authority. Governance, 15(4), 503-529. https://doi.org/10.111 $1 / 1468-0491.00199$ 
Cheng, M., Green, W., Conradie, P., Konishi, N., \& Romi, A. (2014). The international integrated reporting framework: key issues and future research opportunities. Journal of International Financial Management \& Accounting, 25(1), 90-119. https://doi.org/10.1111/jifm.12015

Cooper, D.J., \& Robson, K. (2006). Accounting, professions and regulation: Locating the sites of professionalization. Accounting, Organizations and Society, 31(4), 415-444. https://doi.org/10.1016/j.aos.2006.03.003

Damant D. (2003). Accounting standards - a new era. Balance Sheet, 11(1), 9-20. https://doi.org/10.1108/0965796 0310467836

de Villiers, C, Rinaldi, L., \& Unerman, J. (2014). Integrated Reporting: Insights, gaps and an agenda for future research. Accounting, Auditing \& Accountability Journal, 27(7), 1042-1067. https://doi.org/10.1108/AAAJ-06-2014-1 736

de Villiers, C., Hsiao, P.-C.K. \& Maroun, W. (2017), Developing a conceptual model of influences around integrated reporting, new insights and directions for future research. Meditary Accountancy Research, 25(4), 450-459. htt ps://doi.org/10.1108/MEDAR-07-2017-0183

Deegan, C., Morris, R. \& Stokes, D. (1990). Audit firm lobbying on proposed disclosure requirements. Australian Journal of Management, 15(2), 261-280. https://doi.org/10.1177/031289629001500203

Eccles, R. G., Krzus, M. P., \& Ribot S. (2014). The integrated reporting movement: meaning, momentum, motives, and materiality. Hoboken, NJ: John Wiley \& Sons.

Eccles, R.G., \& Krzus, M.P. (2010). One Report: Integrated Reporting for a Sustainability Strategy. Hoboken, N.J.: John Wiley and Sons.

Fereday, J., \& Muir-Cochrane, E. (2006). Demonstrating rigor using thematic analysis: A hybrid approach of inductive and deductive coding and theme development. International Journal of Qualitative Methods, 5(1), 80-92. http s://doi.org/10.1177/160940690600500107

Flasher, R., Luchs, C. K., \& Souza, J. L. (2018). Sustainability assurance provider participation in standard setting. Research in Accounting Regulation, 30(1), 20-25. https://doi.org/10.1016/j.racreg.2018.03.003

Flower, J. (2015). The international integrated reporting council: a story of failure. Critical Perspectives on Accounting, 27, 1-17. https://doi.org/10.1016/j.cpa.2014.07.002

Fogarty, T. J. (1994). Structural-functionalism and financial accounting: Standard setting in the US. Critical Perspectives on Accounting, 5(3), 205-226. https://doi.org/10.1006/cpac.1994.1012

Fortanier, F., Kolk, A., \& Pinkse, J. (2011). Harmonization in CSR reporting: MNEs and global CSR standards. Management International Review, 51(5), 665-696. https://doi.org/10.1007/s11575-011-0089-9

Georgiou, G. (2004). Corporate lobbying on accounting standards: Methods, timing and perceived effectiveness. Abacus, 40(2), 219-237. https://doi.org/10.1111/j.1467-6281.2004.00152.x

Georgiou, G. (2005). Investigating corporate management lobbying in the UK accounting standard-setting process: A multi-issue/multi-period approach. Abacus, 41(3), 323-347. https://doi.org/10.1111/j.1467-6281.2005.001 85.x

Gibassier, D., Rodrigue, M., \& Arjaliès, D. (2018). Integrated reporting is like God: no one has met Him, but everybody talks about Him. Accounting, Auditing \& Accountability Journal, 31(5), 1349-1380. https://doi.org/10.1108/ AAAJ-07-2016-2631

Giddens, A. (1984). The constitution of society: Outline of the theory of structuration. Berkeley, LA: University of California Press.

Giner, B., \& Arce, M. (2012). Lobbying on accounting standards: Evidence from IFRS 2 on share-based payments. European Accounting Review, 21(4), 655-691. https://doi.org/10.1080/09638180.2012.701796

Hindley, T., \& Buys, P.W. (2012). Integrated reporting compliance with the Global Reporting Initiative framework: An analysis of the South African mining industry. International Business and Economics Research Journal, 11(11), 1249-1260. https://doi.org/10.19030/iber.v11i11.7372 
Humphrey, C., O 'Dwyer, B., \& Unerman, J. (2017). Re-theorizing the configuration of organizational fields: the IIRC and the pursuit of 'Enlightened' corporate reporting. Accounting and Business Research, 47(1), 30-63. https://d oi.org/10.1080/00014788.2016.1198683

International Integrated Reporting Council - IIRC. (2011). Towards Integrated Reporting - Communicating Value in the 21st Century. http://theiirc.org/wp-content/uploads/2011/09/IR-Discussion-Paper-2011_spreads.pdf

International Integrated Reporting Council - IIRC. (2012a). IIRC Governance. http://www.theiirc.org/wp-content /uploads/2011/02/IIRC-GOVERNANCE-2012-04.pdf

International Integrated Reporting Council - IIRC. (2012b). IIRC Due Process. http://www.theiirc.org/wp-conten t/uploads/2012/11/IIRC-Due-Process-25-09-12.pdf

International Integrated Reporting Council - IIRC. (2013a). Consultation draft of the international $<$ IR $>$ framework. http://www.theiirc.org/consultationdraft2013/

International Integrated Reporting Council - IIRC. (2013b). The International <IR> Framework. http://www.theiir c.org/wp-content/uploads/2013/12/13-12-08-THE-INTERNATIONAL-IR-FRAMEWORK-2-1.pdf

International Integrated Reporting Council - IIRC. (2013c). El Marco Internacional $<$ IR>. http://integratedreporting.org/wp-content/uploads/2015/03/13-12-08-THE-INTERNATIONAL-I R-FRAMEWORK-SPANISH-1.pdf

International Integrated Reporting Council - IIRC. (2013d). Future Strategy. Disponible en: http://www.t heiirc.org/wp-content/uploads/2013/12/COUNCIL-20131205-ITEM-4b-STRATEGY.pdf/ [consultado el 20/03/2019]

International Integrated Reporting Council - IIRC. (2013e). Consultation Draft Submissions. http://www.theiirc.o $\mathrm{rg} /$ consultationdraft2013/

International Integrated Reporting Council - IIRC. (2013f). Framework Development: Technical Agenda Papers. ht tp://integratedreporting.org/resource/technical-agenda-papers/

International Integrated Reporting Council (2020a). Consultation Draft. International $<\mathrm{IR}>$ Framework. May 2020. https://integratedreporting.org/wp-content/uploads/2020/05/CONSULTATION-DRAFT_May-21 _IIRC.pdf

Jensen, J.C., \& Berg, N. (2012). Determinants of traditional sustainability reporting versus integrated reporting, an institutionalist approach. Business Strategy and the Environment, 21(5), 299-316. https://doi.org/10.1002/bse .740

Jupe, R.E., (2000). Self-referential lobbying of the Accounting Standards Board: The case of financial reporting standard No. 1. Critical Perspectives on Accounting, 11(3), 337-359. https://doi.org/10.1006/cpac.1999.0366

Kolk, A. (2003). Trends in sustainability reporting by the Fortune Global 250. Business Strategy and the Environment, 12(5), 279-291. https://doi.org/10.1002/bse.370

Kolk, A. (2010). Trajectories of sustainability reporting by MNCs. Journal of World Business, 45(4), 367-374. https: //doi.org/10.1016/j.jwb.2009.08.001

KPMG. (2011). Integrated Reporting Performance insight through Better Business Reporting. Issue 1. http://www.k pmg.com/Global/en/IssuesAndInsights/ArticlesPublications/Documents/road-to-integrated-reporting.pdf

Morros, J. (2016). The integrated reporting: A presentation of the current state of art and aspects of integrated reporting that need further development. Intangible Capital, 12(1), 336-356. https://doi.org/10.3926/ic.700

Nadvi, K., \& Wältring, F. (2004). Making the sense of global standards. En Schmitz, H. (ed.) Local Enterprises in the Global Economy, Issues of Governance and Upgrading (pp. 53-94). Bodmin, OK: Edward Elgar Publishing.

Nölke, A., \& Perry, J. (2007). The power of transnational private governance: Financialization and the IASB. Business and Politics, 9(3), 1-25. https://doi.org/10.2202/1469-3569.1185

Oprisor, T. (2014). The Integrated Reporting Framework: Between Challenge and Innovation. Network Intelligence Studies, II-1(3), 85-94. https://www.ceeol.com/search/article-detail?id=204743 
Paternostro, S. (2020). Integrated reporting and social disclosure: True love or forced marriage? A Multidimensional analysis of a contested concept. En Songini, L. et al. (eds.). Non-Financial Disclosure and Integrated Reporting: Practices and Critical Issues (pp. 107-146). Lance Bingley, UK: Emerald Publishing.

Peters, A., Förster, T., \& Koechlin, L. (2009b). Towards non-state actors as effective, legitimate, and accountable standard setters. En Peters, A. et al. (eds.) Non-state actors as standard setters (pp. 492-562). Cambridge: Cambridge University Press.

Peters, A., Koechlin, L., \& Fenner, G. (2009a). Non-state actors as standard setters: Framing the issue in an interdisciplinary fashion. En Peters, A. et al. (eds.) Non-state actors as standard setters (pp. 1-32). Cambridge: Cambridge University Press.

Reuter, M., \& Messner, M. (2015). Lobbying on the integrated reporting framework: An analysis of comment letters to the 2011 discussion paper of the IIRC. Accounting, Auditing \& Accountability Journal, 28(3), 365-402. http s://doi.org/10.1108/AAAJ-03-2013-1289

Richardson, A. J., \& Eberlein B. (2011). Legitimating Transnational Standard-Setting: The Case of the International Accounting Standards Board. Journal of Business Ethics, 98(2), 217-245. https://doi.org/10.1007/s10551-010 $-0543-9$

Rivera-Arrubla, Y. A. \& Zorio-Grima, A. (2016). Integrated Reporting, Connectivity and Social Media. Journal of Psychology and Marketing, 33(12), 1159-1165. https://doi.org/10.1002/mar.20953

Rivera-Arrubla, Y. A., Zorio-Grima, A. \& García-Benau, M. A. (2016). El concepto de informe integrado como innovación en reporting corporativo. Journal of Innovation \& Knowledge, 1(3), 144-155. https://doi.org/10.1 016/j.jik.2016.01.016

Rivera-Arrubla, Y. A., Zorio-Grima, A. \& García-Benau, M. A. (2017). Integrated Reports: Disclosure level and Explanatory Factors. Social Responsibility Journal, 13(1). https://doi.org/10.1108/SRJ-02-2016-0033

Rowbottom, N., \& Locke, J. (2016). The emergence of <IR>. Accounting and Business Research, 46(1), 86-115. http s://doi.org/10.1080/00014788.2015.1029867.

Sikka, P. (2008). Enterprise culture and accountancy firms: New masters of the universe. Accounting, Auditing \& Accountability Journal, 21(2), 268-295. https://doi.org/10.1108/09513570810854437.

Simnett, R., \& Huggins, A. L. (2015). Integrated reporting and assurance: Where can research add value? Sustainability Accounting, Management and Policy Journal, 6(1), 29-53. https://doi.org/10.1108/SAMPJ-09-2014-0053

Stone, D. (2000). Think Tank Transnationalisation and Non-profit Analysis, Advice and Advocacy. Global Society, 14(2), 153-172. https://doi.org/10.1080/13600820050008421

Suarez, F.F. (2004). Battles for technological dominance: An integrative framework. Research Policy, 33(2), 271-286. https://doi.org/10.1016/j.respol.2003.07.001

Suchman, M. C. (1995). Managing legitimacy: Strategic and institutional approaches. Academy of Management Review, 20(3), 571-610. https://doi.org/10.2307/258788

Suddaby, R., Cooper, D.J., \& Greenwood, R. (2007). Transnational regulation of professional services: Governance dynamics of field level organizational change. Accounting, Organizations and Society, 32(4), 333-362. https://d oi.org/10.1016/j.aos.2006.08.002

Thomson, I. (2015). But does sustainability need capitalism or an integrated report' a commentary on 'The International Integrated Reporting Council: A story of failure' by Flower, J. Critical Perspectives on Accounting, 27, 18-22. https://doi.org/10.1016/j.cpa.2014.07.003

Tutticci, I., Dunstan, K., \& Holmes, S. (1994). Respondent lobbying in the Australian accounting standard-setting process: ED49-a case study. Accounting, Auditing \& Accountability Journal, 7(2), 86-104. https://doi.org/10.1 $108 / 09513579410058201$

Tweedie, D., \& Martinov-Bennie, N. (2015). Entitlements and Time: Integrated Reporting's Double-edged Agenda. Social and Environmental Accountability Journal, 35(1), 49-61. https://doi.org/10.1080/0969160X.2015.100 7466

Vogel, D. (2008). Private global business regulation. Annual Review of Political Science, 11, 261-282. https://doi.org /10.1146/annurev.polisci.11.053106.141706 
Zeff, S. A. (1999). La evolución del marco conceptual para las empresas mercantiles en Estados Unidos. Revista Española de Financiación y Contabilidad, 151-194. https://dialnet.unirioja.es/servlet/articulo?codigo $=44297$

Zicari, A. (2014). Can One Report be Reached? The Challenge of Integrating Different Perspectives on Corporate Performance. En Tench et al. (eds). Communicating Corporate Social Responsibility: Perspectives and Practice Critical Studies on Corporate Responsibility, Governance and Sustainability (vol. 6, pp. 201-216). Lance Bingley, UK: Emerald Group Publishing Limited.

\section{Notas}

* Artículo de investigación.

1 Los números de referencia indicados como "ref." corresponden a la codificación asignada por el IIRC a las comunicaciones recibidas en respuesta al CD de 2013. Todas las cartas de comentarios y cuestionarios enviados por los participantes pueden consultarse en Submissions to the Consultation Draft of The International <IR $>$ Framework en el sitio web del IIRC (IIRC, 2013e).

2 http://www.theiirc.org/crd/crd-for-web-2/

\section{Licencia Creative Commons CC BY 4.0}

Para citar este artículo: Rivera-Arrubla, Y. A. (2021). El papel de los organismos no estatales de estandarización en la formulación del Marco Internacional de Información Integrada. Cuadernos de Contabilidad, 22. https://doi.org/10.11144/Javeriana.cc22.poee 